\title{
Alternativen beim Pflanzenschutz?
}

\author{
Kurt Mendgen \\ Lehrstuhl Phytopathologie, Fakultät für Biologie der Universität, D-7750 Konstanz
}

The control of plant diseases may be improved by influencing the development of a parasite in its host. The role of elicitors and inducers for the induction of the plant's defense reactions and the use of hyperparasites is discussed as a part of an integrated pest management system of rust fungi.

Zur Bekämpfung von Schaderregern stehen dem Landwirt die unterschiedlichsten Methoden zur Verfügung: allgemeine, pflanzenbauliche Maßnahmen [1], biologische Pflanzenpflegemittel (die doch nicht mehr helfen als eine Seifenlösung), der Pflanzenschutz des biologisch dynamischen Landbaus [2] oder der moderne chemische Pflanzenschutz [3]. Sie werden hier nicht behandelt. Stattdessen sollen (oft noch nicht praxisreife) umweltschonende Methoden dargestellt werden, die man aus einer genauen Kenntnis von Wirt-Parasit-Systemen ableiten kann. Als Beispiel werden die Rostpilze herausgegriffen. Diese zu den Basidiomyceten (Unterklasse Phragmobasidiomycetes, Ordnung Uredinales) gehörende Pilzgruppe befällt fast alle Kulturpflanzen. Man erkennt sie an den oft rostfarbigen Pusteln auf den Blättern, so auch den Bohnenrost (Uromyces appendiculatus var. appendiculatus). Im Gegensatz zu den bei uns mehr bekannten Getreiderostpilzen ist der Bohnenrost eher an mediterrane Bedingungen angepaßt. Besonders in Lateinamerika und Australien, wo er sich das ganze Jahr über vermehren kann, ruft er große Schäden an allen Phaseolus-Bohnen hervor, indem er die Bohnenhülsen mit großen zimtbraunen Sporenlagern bedeckt. Wie die meisten Rostpilze hat auch der Bohnenrost einen recht komplizierten Entwicklungsverlauf mit fünf Sporenformen, jeweils mit einer bestimmten Kernphase gekoppelt [4]. Für die weiträumige Verbreitung des Pilzes ist die Uredospore verantwortlich. Sie kann schon fünf bis acht Tage nach Infektion ein Sporenlager hervorbringen, das viele Millionen Sporen produziert, die wie brauner Staub aus den Blättern quellen. Bis etwa 1850 glaubten viele Wissenschaftler, daß dies die kranken Säfte der Pflanzen seien, die sich an der Luft zu diesem Staub umformten. Julius Kühn (1858) und Anton De Bary (1884) waren mit die ersten Forscher [5], die die Keimung der Sporen beobachteten, ihr Eindringen in das Blatt verfolgten und sich Gedanken über die Entwicklung und Vermehrung der Parasiten machten.

\section{Der Parasit paßt sich der Wirtspflanze an}

Wenn eine Rostspore auf Wasser fällt, keimt sie. Der Keimschlauch stellt sein Wachstum nach etwa $10-20 \mathrm{~h}$ ein und stirbt bald darauf. Nährstoffzusätze helfen in der Regel wenig [6]. Wenn die Rostpore jedoch auf ein Blatt einer Wirtspflanze fällt und die Luft bei $16-22^{\circ} \mathrm{C}$ mindestens $8 \mathrm{~h}$ lang wassergesättigt bleibt, geschieht folgendes: Der Keimschlauch wächst quer zu den Antiklinen der Epidermiszellen und findet mit großer Wahrscheinlichkeit eine Spaltöffnung. Bei Nichtwirtpflanzen, je nach Struktur der Blattoberfläche, findet der Bohnenrost die Spaltöffnung entsprechend seltener [7]. Die Vorhofleisten der Schließzellen üben einen mechanischen Reiz auf die Hyphenspitzen aus: Der Pilz differenziert ein Appressorium über der Spaltöffnung aus (Fig. 1a). Dieses Gebilde ist eine Art Haftorgan, mit dem sich der Rostpilz über der Spaltöffnung verhakt [8]. Jetzt entwickelt sich auf dem Boden des Appressoriums der Infektionskeil (Fig. 1 b, c), der die Schließzellen auseinanderdrückt; nun kann der Pilz in die Atemhöhle hineinwachsen. In der Atemhöhle des Blattes entsteht das substomatäre Vesikel, in dem sich das gesamte Plasma des Pilzes ansammelt (Fig. 1d, e). Der Rostpilz benötigt für diesen Vorgang etwa 


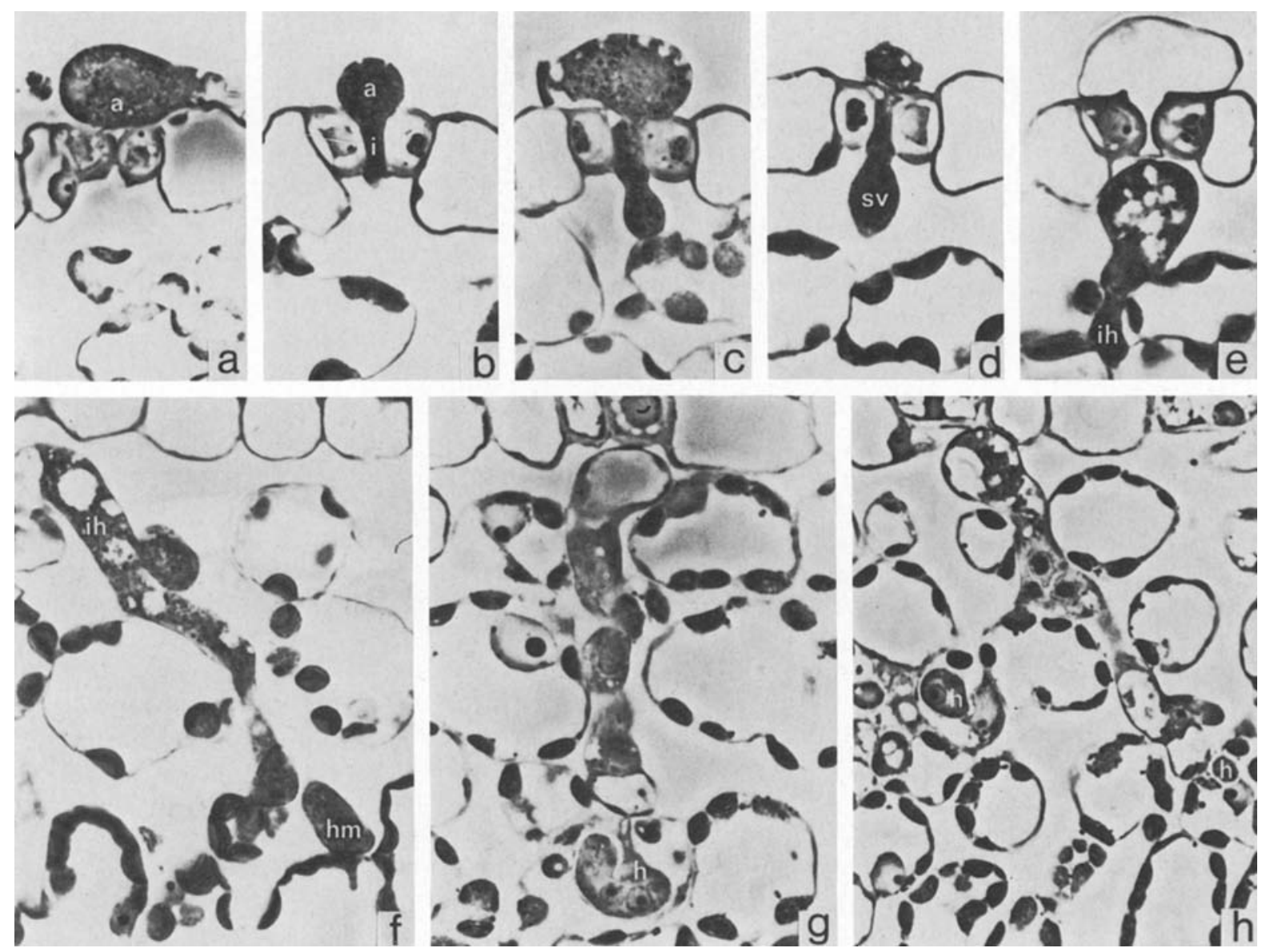

Fig. 1. a) Der Keimschlauch des Bohnenrosts ( $U$. appendiculatus) hat die Spaltöffnung gefunden und über den Schließzellen das Appressorium (a) ausdifferenziert. b) Auf dem Boden des Appressoriums (Querschnitt) entsteht der Infektionskeil (i). c) Längsschnitt durch das Appressorium mit Infektionskeil. d) In der Atemhöhle bildet sich das substomatäre Vesikel (sv). e) Aus dem substomatären Vesikel wachsen Infektionshyphen (ih). f) In der Mitte des Blattparenchyms differenziert die Infektionshyphe (ih) die HaustorienMutterzelle $(\mathrm{hm})$ aus, die mit dem Penetrationskeil in die Zelle eindringt. g) Es entsteht das Haustorium $(h)$. h) Die Hyphen verzweigen sich, und es werden weitere Haustorien ausgebildet $(\times 1100$, aus [9])

6-10 $\mathrm{h}$ und ist von nun an von den Außenbedingungen in der Umgebung der Pflanze unabhängig. Aus dem substomatären Vesikel wachsen Infektionshyphen, die sich an die Parenchymzellen anschmiegen. Der Kontakt der Infektionshyphe mit der Wand der Parenchymzelle scheint die Ausdifferenzierung der Haustorien-Mutterzelle (Fig. 1f) auszulösen: Wandmaterial der Hyphen haftet besonders gut an Zellwänden von Wirtspflanzen, und nur die Wände der Parenchymzellen induzieren die Ausbildung der Haustorien-Mutterzelle [9, 10]. Nur die Haustorien-Mutterzelle kann das Haustorium ausbilden (Fig. $1 \mathrm{~g}$ ), ein keulenförmiges Gebilde in der Wirtszelle, das jedoch das Plasmalemma nicht durchstößt, sondern nur eindellt. Gleichzeitig wird das Plasmalemma auf charakteri- stische Weise modifiziert [11]. Der schmale Teil dieser Keule wird Hals des Haustoriums genannt: Hier wird nach Osmiumsäure-Behandlung ein Halsring beobachtet, dem man ähnliche Funktionen wie dem Casparyschen Streifen in der Wurzel zuschreibt [12]. Mit radioaktiv markierten Metaboliten wurde die Nahrungsaufnahme über die Haustorien bewiesen [13]. Die Pilzhyphen verzweigen sich jetzt (Fig. 1 h), und schon nach 5-8 Tagen hat sich ein dichtes Mycel im Interzellularraum ausgebildet, welches die Uredosporen abschnürt. Die Sporen zerdrücken einen Teil des Parenchyms, die Epidermis platzt auf, und die Sporen häufen sich - wie rostiger Staub - auf der Blattoberfläche an. Es entsteht die charakteristische Rostpustel. 


\section{Störung der Anpassung zwischen Wirt und Parasit}

Die fein abgestimmte Anpassung des Rostpilzes an die Wirtspflanze muß nun bei der Bekämpfung des Schaderregers gestört werden: So züchtet der praktische Züchter seine Kulturpflanze auf Resistenz, indem er einfach die Pflanzen in seinem Zuchtgarten ausliest, die einen geringeren Befall zeigen. Dieser geringere Befall hat dann seine Gründe, z.B. ist ein Gen bei der Gerste bekannt, das die Oberflächenstruktur der Cuticula, des Deckhäutchens der Blattoberfläche, verändert [14]. Dann erkennt der Keimschlauch des Mehltaus (Erysiphe graminis) die Wirtspflanze nicht mehr, und der Infektionsvorgang wird unterbrochen. Bei Rostpilzen wurde noch keine mutierte Wirtspflanze gefunden, die ihre Oberfläche so verändert hätte oder ihre Spaltöffnung so fest schließt, daß der Rost nicht eindringen könnte. Es sind aber inzwischen viele Bohnen gezüchtet worden, die bei der Ausbildung des Haustoriums den weiteren Infektionsverlauf des Pilzes unterbrechen. Eine besonders auffällige Abwehrreaktion der Zelle besteht in der totalen Abkapselung eines Haustoriums durch die Zelle mit einem Kallose-ähnlichen Material [15]. Bei der Bohnensorte Golden Gate Wax ist das Haustorium zu keiner normalen Metabolit-Aufnahme in der Lage [16], und bei der Bohnensorte 017 erlaubt die Wirtspflanze zunächst ein normales Wachstum aber nach drei Tagen sterben die Parenchymzellen am Infektionsort ab: Mit bloßem Auge erkennt man eine nekrotische Stelle auf dem Blatt, wir sprechen dann vom hypersensitiven Zelltod oder von Hypersensitivität. Die infizierte Blattregion stirbt nicht nur $a b$, die Wirtszellen produzieren außerdem für den Pilz giftige Substanzen, sogenannte Phytoalexine [17].

In der Praxis war es eine große Enttäuschung, als sich herausstellte, daß viele resistente Pflanzen nach einigen Jahren im Anbau wieder anfällig wurden. Wir können dies heute, trotz noch bestehender Kontroversen, einigermaßen verstehen: Durch die vom Züchter selektierte Veränderung der Wirtspflanze gerät die Abstimmung zwischen Wirtspflanze und Parasit in Unordnung. Im praktischen Anbau selektiert jedoch die resistente Pflanze solche Parasitenrassen, bei denen diese Abstimmung wieder paßt. Dieses System ist dann kompatibel, d.h. die Pflanze ist wieder anfällig. Ziel der Forschung ist es jetzt, herauszufinden, warum einige resistente Kultursorten über Jahrzehnte hinweg resistent geblieben sind, andere dagegen schon nach wenigen Generationen wieder anfällig wurden. Wir wissen nur, daß Pflanzen, die das Wachstum eines Parasiten zwar behindern, aber nicht völlig ausschalten, oft länger resistent bleiben. Im Rahmen komplizierter Gen-Management-Programme versuchen die Züchter nun, diese Gene im Rahmen von sorgfältig ausgearbeiteten Sortenanbau-Programmen so einzusetzen, daß die Resistenz-Eigenschaften der Kultursorten möglichst lange erhalten bleiben [18].

\section{Eingriff in die Regulation der Wirt-Parasit-Beziehungen}

Wir verstehen den Wirkungsmechanismus der Resistenz-Gene also noch nicht. Wenn wir hier Fortschritte erzielten, könnten wir dem Züchter vielleicht Hinweise geben, wie er züchten soll, damit eine jahrelange Arbeit nicht nach kurzer Zeit wieder wertlos wird. Es gibt jedoch neue interessante Aspekte: Bushnell und Rowell [19] schlagen vor, daß das Wachstum der Parasiten in der Pflanze durch Elicitoren und Suppressoren geregelt wird. Die Elicitoren rufen die Abwehrreaktionen bei Pflanzen hervor, die Suppressoren unterdrücken diese Abwehrreaktionen. Ein optimal adaptierter Parasit würde also neben dem Elicitor, den alle Pilze besitzen, den effektivsten Suppressor produzieren. Heath [20] unterteilt diese Möglichkeiten der Interaktion zwischen Wirt und Parasit noch feiner: Sie unterscheidet zwischen WirtspflanzenSpezifität und Sorten-Spezifität. Bei der Wirtspflanzen-Spezifität hat sich der Erreger an eine Wirtspflanze so eng angepaßt, daß er nicht, wic alle anderen Organismen, durch unspezifische $A b$ wehrmechanismen der Wirtszellen abgewehrt wird. So beherbergen viele Pflanzen präformierte toxische Substanzen, die während einer Infektion durch einen Parasiten freigesetzt werden können [21]. Der Erreger kann diese Vorgänge nun mit dem Suppressor unterdrücken, er kann dagegen unempfindlich sein, oder er kann toxische Produkte der Wirtspflanze einfach abbauen. Es entsteht also eine Basisverträglichkeit der Wirtspflanze gegenüber dem Parasiten. Die Sorten-Spezifität beeinflußt die Feinabstimmung zwischen Wirt und Rassen des Parasiten: Der Züchter selektiert sehr oft (für den Parasiten) geringfügige $\mathrm{Mu}$ tationen bei der Wirtspflanze, die der angepaßte Parasit durch entsprechende geringfügige Mutation wieder überwinden kann. Diese Veränderungen, die die Sorten-Spezifität beeinflussen, betreffen also nur das jeweilige Wirt-Parasit-System und nicht allgemeine Abwehrmechanismen der Pflanze. Es bleibt offen, ob diese theoretisch unterscheidbaren Möglichkeiten der Wirtspflanze im Experiment nachweisbar sein werden. Es müßten sich dann 
spezifische und unspezifische Elicitoren und Suppressoren nachweisen lassen.

Eine Reihe von Forschergruppen haben diese Elicitoren und Suppressoren bisher untersucht und, wenn auch nur recht grob, gereinigt und isoliert. Elicitoren sind Glykoproteine oder, im einfachsten Falle, Glucane, die sich hauptsächlich aus Pilzwänden isolieren lassen und recht unspezifisch wirken. Sie induzieren in der Pflanzenzelle die Synthese der Phytoalexine [17]. Wenn man Elicitoren aus Keimschläuchen des Bohnenrosts in den Interzellularraum des Bohnenblattes infiltriert, beobachtet man wenige Tage später eine Anreicherung von Phytoalexinen in diesen Blättern und, wenn man diese mit Bohnenrost infiziert, eine drastische Reduktion der Pustelzahl [22]. Ablagerungen auf der Zellwand scheinen die Ausbildung von Haustorien zu behindern [23]. Andererseits lassen sich aus Rostinfiziertem Gewebe und aus Rostpilz-Hyphen Suppressoren, deren chemische Charakterisierung noch aussteht, gewinnen. Diese Suppressoren unterdrücken die Ausbildung von Silicium-verstärkten Ablagerungen auf der Zellwand, die man beobachtet, wenn der Rost der Kuhbohne. (Uromyces phaseoli var. vignae) in die Parenchymzelle der Bohne (Phaseolus vulgaris) einzudringen versucht [24]. Rassenspezifisch wirkende Elicitoren und Suppressoren wurden bei Rostpilzen anscheinend noch nicht gefunden, wohl aber bei anderen WirtParasit-Systemen [25].

Obwohl diese Ergebnisse zumindest teilweise noch etwas vorläufig sind, scheint es doch möglich zu sein, von außen in die Regulation von Wirt-Parasit-Systemen einzugreifen. Wenn es wirklich gelingen würde, ungiftige Substanzen als Pflanzenschutzmittel auszubringen, die nur unspezifische Abwehrmechanismen in der Pflanze aktivieren, wäre dies ein großer Erfolg. Man würde hierbei nicht die Sorten-Spezifität beeinflussen, die sich nur auf ein bestimmtes Wirt-Parasit-System bezieht, sondern könnte unterschiedliche Parasiten gleichzeitig bekämpfen. Bisher wurden folgende Wege bei praktischen Versuchen beschritten: Ward et al. [26] beobachteten, daß Applikation des Fungizids Metalaxyl [Wirkstoff $( \pm)$-Methyl2-N-(2-methoxyacetyl)-2,6-xylidino-propionat] die Phytoalexin-Synthese bei der Sojabohne deutlich steigert. Zuerst wurde dies als Effekt des Fungizids angesehen, welches die Synthese der für den Pilz toxischen Substanzen anregt [27]. Neuerdings neigen viele Experimentatoren jedoch mehr zu der Ansicht, daß Wandbestandteile des toten Pilzes im Gewebe der Pflanze diese Phytoalexin-Produktion anregen [28]. Schönbeck et al. [29] berichten über die Isolierung von Substanzen aus Nährlösungen verschiedener Mikroorganismen, die sie Induktoren nennen. Die Zusammensetzung und die Struktur dieser Induktoren sind noch unbekannt. Bei grob vorgereinigten Präparaten reichte das Besprühen der Pflanzen aus, um für mehrere Tage einen Schutz vor Rostpilzen, Echten und Falschen Mehltaupilzen, herbeizuführen. Vorläufige Experimente im Freiland zeigten günstige Resultate bei Gurken und Getreide, die von Echtem Mehltau befallen waren. Im Vergleich dazu war die Wirkung der derzeit üblichen Fungizide nur wenig besser [30].

\section{Der Einsatz von Hyperparasiten}

Es ist naheliegend, bei der Bekämpfung eines Parasiten an die Antagonisten dieses Parasiten zu denken. Hier können nicht alle Möglichkeiten, die bisher erfolgreich angewandt wurden, aufgezählt werden. Es gibt Beispiele für Insekten [31], Unkräuter [32] und Pilze [33]. Bei pflanzenpathogenen Pilzen ist dies jedoch nicht so einfach. Im Gegensatz zu Mikroorganismen, die Antibiotika ausscheiden, oder Insekten, die ihren Wirt aktiv aufsuchen, sind Pilze relativ unbeweglich. Pilzparasiten benötigen einen direkten Kontakt mit dem zu parasitierenden Pilz. Dabei kann es sich um eine Konkurrenz ums Substrat, antagonistische Effekte oder um einen direkten Parasitismus handeln, bei dem der befallene Pilz in der Folge abstirbt. Besonders erfolgversprechend sind Methoden, bei denen ein Pilz die Dauerstadien pflanzenpathogener Pilze, wie die Sklerotien oder die Teleutosporen, befällt. Dann hat der Hyperparasit während des Winters genügend Zeit und oft auch günstige Bedingungen, um diese Dauerformen abzutöten [34].

Der Einsatz von Hyperparasiten zur Bekämpfung von Pilzen auf oberirdischen Teilen der Pflanze ist jedoch ungleich schwieriger. Die Triebe und Blätter der Pflanze sind sehr unterschiedlichen Temperaturen und Luftfeuchtigkeiten ausgesetzt [33]. Die Sonneneinstrahlung ist ungünstig für Pilzhyphen. Der Regen wäscht die Sporen $a b$, und die Oberfläche der Pflanze ist oft so ausgeprägt, daß sie für die meisten Pilze ein ungünstiges Substrat darstellt. Der Hyperparasit, der ja auf den pflanzenpathogenen Pilz spezialisiert ist, die Pflanze selbst aber nicht befallen soll, muß eine hohe Vermehrungsrate besitzen und persistent bei ungünstigen Bedingungen sein, damit er den Pflanzenparasiten auch auffinden und schneller bekämpfen kann, als dieser sich ausbreitet. In der Praxis wird man meist ein Gleichgewicht zwischen Hyperparasit und Parasit beobachten, das der Landwirt immer wieder in die gewünschte Richtung beeinflussen muß. So parasitiert Verticillium lecanii die Sporenlager vieler 

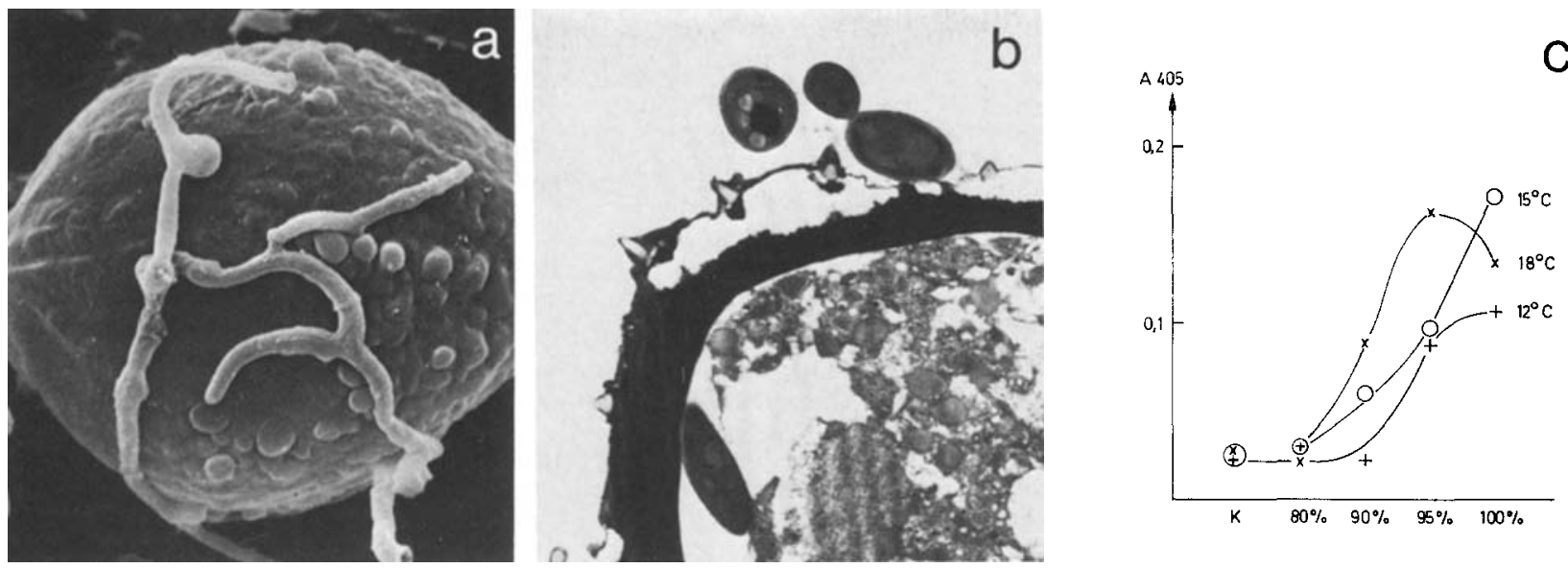

Fig. 2. a) Hyphen von V. lecanii auf einer Teleutospore von U. appendiculatus ( $\times 2000$, Aufnahme Ch. Grabski). b) Querschnitt durch eine von $V$. lecanii befallene Uredospore. Das Wandmaterial der Spore ist teilweise abgebaut $(\times 2000)$. c) Einfluß von Temperatur und Luftfeuchte auf das Wachstum von V. lecanii in Pusteln des Gelbrosts (Puccinia striiformis), gemessen mit dem ELISA-Test (enzyme-linked immunosorbent assay) (aus [38])

Rostpilze [35], Blattläuse und Schildläuse [36]. Anscheinend ist dieser Pilz mit einer sehr effektiven Chitinase ausgerüstet, mit der er die Chitin-haltige, dicke Sporenwand von Rostpilzen und die Cuticula der Insekten auflösen kann. Das Blattgewebe der Wirtspflanze und das Mycel des Rostpilzes im Gewebe werden zunächst nicht angegriffen [37]. Der Hyperparasit konzentriert sich auf die Vermehrungsform des Rostpilzes, die Sporen (Fig. 2a, b). Die Wachstumsbedingungen für den Rostpilz und den Hyperparasiten sind jedoch unterschiedlich: Während der Rostpilz nur während des Keimschlauchwachstums, also bis zur Ausbildung des Appressoriums, eine wassergesättigte Atmosphäre benötigt, weil das weitere Wachstum im Blattinnern erfolgt, benötigt der Hyperparasit $V$. lecanii konstant hohe Luftfeuchtigkeiten (Fig. 2c). Eine Abhilfemöglichkeit besteht darin, die Blätter mit Stärke oder anderen hygroskopischen Kohlehydraten zu besprühen, damit $V$. lecanii möglichst günstige Bedingungen zum Wachstum auf der Blattoberfläche vorfindet.

Ein ähnliches Problem tritt beim Einsatz des Hyperparasiten Ampelomycis quisqualis zur Bekämpfung des Gurkenmehltaus (Sphaerotheca fuliginea) auf. Wenn die Gurken aber im Gewächshaus angezogen werden, kann man hier durch regelmäßiges Sprühen [39] dem Hyperparasiten die nötigen günstigen Bedingungen anbieten.

\section{Welche Probleme gibt es in der Praxis?}

Diese Ergebnisse sind nicht nur wissenschaftlich interessant, sondern auch vielversprechend für die
Praxis. Der Eingriff in die Regulation der WirtParasit-Beziehungen und der Einsatz von Hyperparasiten scheinen durchführbar zu sein. Bei der Bekämpfung von Insektenplagen werden Mikroorganismen schon vielfach mit Erfolg angewendet [31]. Grundsätzlich sind hier jedoch folgende Bemerkungen notwendig (für spezielle Fälle s. [40]): Substanzen ,biologischen“" Ursprungs oder Mikroorganismen sind per se nicht weniger toxisch als die üblichen Fungizide (oder Insektizide o.ä.). Jahrelange Toxizitätsuntersuchungen sind also auch hier notwendig. Die zulassende Behörde, die Biologische Bundesanstalt für Land- und Forstwirtschaft, steht vor der schwierigen Frage, ob sie biologischen Methoden einen gewissen „Bonus“ gibt, damit sie schneller eingesetzt werden können.

Die Infektiosität des eingesetzten Organismus gegenüber Mensch, Tier und ,nützlichen“ Pflanzen ist genau zu überprüfen. Die Möglichkeit, Toxine $\mathrm{zu}$ produzieren, Allergien hervorzurufen oder genetische Informationen zu übertragen, muß sorgfältig untersucht werden.

Diese neuen Mittel müssen in der Praxis etwa gleich wirksam sein wie die üblichen Fungizide und wirtschaftlich eingesetzt werden können. Sonst werden sie von der Praxis nicht angenommen.

Trotz aller Einschränkungen ist also damit zu rechnen, daß wir in Zukunft mit neuen, umweltschonenden Pflanzenschutzmethoden rechnen können. In der Praxis wird es notwendig sein, diese Ergebnisse in die althergebrachten Pflanzenschutzsysteme einzubauen. Eine Kombination von pflanzenbaulichen Maßnahmen, Prognose-Verfahren 
zur sparsamen Anwendung von Pflanzenschutzmitteln und der neuen, hier geschilderten Methoden scheint möglich zu sein.

In den USA hat man dafür schon frühzeitig den Begriff ,,integrated pest management" geprägt. Dieser ,,integrierte Pflanzenschutz" wird also noch komplizierter werden.

An vielen Universitäten der USA gibt es inzwischen Lehrprogramme für integrierte Pflanzenschutzsysteme. Für spezielle Schaderreger gibt es ausgearbeitete Programme zur biologischen Schädlingsbekämpfung [41]. Bei uns versucht die Deutsche Phytomedizinische Gesellschaft, im Rahmen eines Aufbaustudiums und einer Fachprüfung diese Situation zu verbessern. Aber auch der Gesetzgeber könnte regulierend eingreifen, um den Einsatz hochgiftiger Pflanzenschutzmittel zu reduzieren: Mir ist nicht einleuchtend, warum ein Kind im ,Laden an der Ecke ${ }^{\text {“ }}$ Pflanzenschutzmittel kaufen kann, wovon wenige mg oder g tödlich sein können.

1. Heitefuss, R.: Pflanzenschutz, Grundlagen der praktischen Phytomedizin. Stuttgart: Thieme 1975

2. Alternativen zum gegenwärtigen Landbau. Frankfurt: DLG-Verlag 1980

3. Buchenauer, H., in: The Rust Fungi (Scott, K.J., Chahavorty, A.K., eds.). New York: Academic Press 1982

4. Müller, E., Loeffler, W.: Mykologie. Stuttgart: Thieme 1982

5. Kühn, J.: Die Krankheiten der Kulturgewächse, ihre Ursachen und ihre Verhütung. Berlin: Bosselmann 1858; De Bary, A.: Vergleichende Morphologie und Biologie der Pilze, Mycetozoen und Bacterien. Leipzig: Engelmann 1884

6. Fuchs, W.H., Gaertner, A.: Arch. Mikrobiol. 28, 303 (1958)

7. Heath, M.C.: Physiol. Plant Pathol. 4, 403 (1974)

8. Mendgen, K.: Phytopath. Z. 78,109 (1973)

9. Mendgen, K.: ibid. 93, 295 (1978)

10. Mendgen, K.: Arch. Microbiol. 119, 113 (1978); Mendgen, K. : Naturwissenschaften 69, 502 (1982)

11. Harder, D.E., Mendgen, K. : Protoplasma 112, 46 (1982)

12. Heath, M.C.: Can. J. Bot. 54, 2482 (1976); Chong, J., Harder, D.E.: ibid. 58, 2496 (1980)

13. Mendgen, K.: Phytopathology 71, 983 (1981)

14. Yang, S.L., Ellingboe, A.H.: ibid. 62,708 (1972)

15. Heath, M.C.: ibid. 61, 383 (1971)

16. Mendgen, K.: Naturwissenschaften 64, 438 (1977)

17. Elnaghy, M.A., Heitefuss, R.: Physiol. Plant Pathol. 8, 269 (1976); Grisebach, H., Ebel, J.: Angew. Chem. 90, 668
(1978); Hoppe, H.H.: Qual. Plant, Plant Foods Hum. Nutr. 30, 289 (1981); Bailey, J.A., Mansfield, J.W. (eds.): Phytoalexins. Glasgow: Blackie 1982

18. Wolfe, M.S., Barret, J.A.: Plant Disease 64, 148 (1980); Zadoks, J.C., Schein, R.D.: Epidemiology and Plant Disease Management. Oxford 1979

19. Bushnell, W.R., Rowell, J.B.: Phytopathology 71, 1012 (1981)

20. Heath, M.C.: ibid. 71, 1121 (1981)

21. Schlösser, E.W., in: Plant Disease, Vol. 5 (Horsfall, J.G., Cowling, E.B., eds.). New York: Academic Press 1980; Schönbeck, F., in: Specificity in Plant Diseases (Wood, R.K.S., Graniti, A., eds.). New York-London: Plenum Press 1976

22. Hümme, B., Hoppe, H.H., Heitefuss, R.: Phytopath. Z. 92, 281 (1978); Hoppe, H.H., Hümme, B., Heitefuss, R.: ibid. $97,85(1980)$

23. Ebrahim-Nesbat, F., Hoppe, H.H., Heitefuss, R.: ibid. 103, 261 (1982)

24. Heath, M.C.: Physiol. Plant Pathol. 18, 149 (1981)

25. Ziegler, E., Pontzen, R.: ibid. 20, 321 (1982)

26. Ward, E.W.B., et al.: Phytopathology 70, 738 (1980)

27. Cartwright, D., et al.: Nature 267, 511 (1977)

28. Stössel, P., Lazarovits, G., Ward, E.W.B.: Phytopathology $72,106(1982)$

29. Schönbeck, F., Dehne, H.W., Beicht, W.: Z. Pflanzenkrankh. Pflanzensch. 87, 654 (1980)

30. Beicht, W.: Dissertation Univ. Hannover 1981; Schönbeck, F., Dehne, H.W., Balder, H.: Z. Pflanzenkrankh. Pflanzensch. 89, 177 (1982)

31. Franz, J.M., Krieg, A.: Biologische Schädlingsbekämpfung. Berlin-Hamburg: Parey 1976

32. Templeton, G.E.: Weed Sci. 30, 430 (1982)

33. Burges, H.D.: Microbial Control of Pests and Plant Diseases 1970-1980. London: Academic Press 1981; Kranz, J., in: Microbial Ecology of the Phylloplane (Blackman, G.P., ed.). New York: Academic Press 1981

34. Mangenot, F., Diem, H.G., in: Ecology of Root Pathogens (Krupa, S.V., Dommergues, Y.R., eds.). Amsterdam: Elsevier 1979

35. Hassebrauk, K.: Phytopath. Z. 9, 513 (1936); Mendgen, K.: Mitt. Biol. Bundesanst. 191, 301 (1979)

36. Hall, R.A., in: Microbial Control of Pests and Plant Diseases 1970-1980 (Burges, H.D., ed.). London: Academic Press 1981

37. Mendgen, K., Casper, R.: Phytopath. Z. 99, 362 (1980)

38. Mendgen, K.: ibid. 102, 301 (1981)

39. Philipp, W.D., Krüger, G. : Z. Pflanzenkrankh. Pflanzensch. $86,129(1979)$

40. Krieg, A.: Nachrichtenbl. dtsch. Pflanzenchutzd. 30, 177 $(1978) ; 33,5(1980)$

41. Goldstein, J. (ed.): The Least Is Best Pesticide Strategy. Emmaus, P.A.: G. Press 1978; Mendgen, K.: Neue Wege im Pflanzenschutz. Konstanz: Universitätsverlag 1981

Eingegangen am 13. September 1982 